\title{
Fine-scale features on the sea surface in SAR satellite imagery - Part 2: Numerical modeling
}

\author{
S. Matt ${ }^{1, *}$, A. Fujimura ${ }^{2}$, A. Soloviev ${ }^{1,2}$, S. H. Rhee $^{3}$, and R. Romeiser ${ }^{2}$ \\ ${ }^{1}$ Nova Southeastern University Oceanographic Center, Dania Beach, FL, USA \\ ${ }^{2}$ University of Miami Rosenstiel School of Marine and Atmospheric Science, Miami, FL, USA \\ ${ }^{3}$ Seoul National University, Department of Naval Architecture \& Ocean Engineering, Seoul, South Korea \\ * now at: NRC Research Associate at Naval Research Laboratory, Stennis Space Center, MS, USA
}

Correspondence to: S. Matt (silvia.matt.ctr@nrlssc.navy.mil, silvia.matt@nova.edu)

Received: 30 June 2012 - Published in Ocean Sci. Discuss.: 17 September 2012

Revised: 7 March 2014 - Accepted: 11 March 2014 - Published: 2 June 2014

\begin{abstract}
With the advent of the new generation of synthetic aperture radar (SAR) satellites, it has become possible to resolve fine-scale features on the sea surface on the scale of meters. The proper identification of sea surface signatures in SAR imagery can be challenging, since some features may be due to atmospheric distortions (gravity waves, squall lines) or anthropogenic influences (slicks), and may not be related to dynamic processes in the upper ocean. In order to improve our understanding of the nature of fine-scale features on the sea surface and their signature in SAR, we have conducted high-resolution numerical simulations combining a three-dimensional non-hydrostatic computational fluid dynamics model with a radar imaging model. The surface velocity field from the hydrodynamic model is used as input to the radar imaging model. The combined approach reproduces the sea surface signatures in SAR of ship wakes, low-density plumes, and internal waves in a stratified environment. The numerical results are consistent with observations reported in a companion paper on in situ measurements during SAR satellite overpasses. Ocean surface and internal waves are also known to produce a measurable signal in the ocean magnetic field. This paper explores the use of computational fluid dynamics to investigate the magnetic signatures of oceanic processes. This potentially provides a link between SAR signatures of transient ocean dynamics and magnetic field fluctuations in the ocean. We suggest that combining SAR imagery with data from ocean magnetometers may be useful as an additional maritime sensing method. The new approach presented in this work can be extended to other dynamic processes in the upper ocean, including fronts and eddies, and
\end{abstract}

can be a valuable tool for the interpretation of SAR images of the ocean surface.

\section{Introduction}

Synthetic aperture radar (SAR) imaging of the sea surface has long been recognized to be an important tool for ship surveillance, as well as oil spill and pollution monitoring. SAR satellite imagery may be used to gain information about dynamic processes in the upper ocean. Large amplitude internal waves are a well-documented example (Alpers, 1985; Hsu et al., 2000; Alpers et al., 2008, and others). Furthermore, under certain conditions, currents and winds can also be inferred from the new generation of SAR satellites (Horstmann et al., 2000; Romeiser et al., 2001, 2010; Brusch et al., 2008). However, atmospheric distortions and anthropogenic influences may complicate the interpretation of SAR satellite imagery (Alpers, 1985; Soloviev et al., 2012). Surfactants on the sea surface may also play an important role in the visibility of fine-scale features on the sea surface in SAR imagery (Hühnerfuss et al., 1987; Gade et al., 1998).

To improve our understanding of the characteristics of sea surface signatures of fine-scale oceanic features in SAR images, we have combined a three-dimensional non-hydrostatic computational fluid dynamics (CFD) model with a radar imaging model. This computational approach was first employed by Fujimura et al. (2010) to the study of the turbulent centerline wake of a ship. 
In this paper, we apply their approach to the study of three types of oceanic phenomena, ship wakes, low-density buoyancy-driven currents, and internal wave solitons. The hydrodynamics of far wakes of ships with application to SAR remote sensing have previously been studied by various authors (Milgram et al., 1993a, b; Reed and Milgram, 2002; Soloviev et al., 2008, 2010), and it is a topic that has important practical applications. These include coastal and fishery management, ship surveillance and identification, as well as pollution and environmental monitoring (Pichel et al., 2004). Fujimura et al. (2010) found that wind stress across the ship wake leads to an asymmetry in the wake surface signature in simulated radar images. Here, we use their model of the ship wake and extend the analysis to include an ambient thermal stratification to investigate the impact of a stratified water column on the surface characteristics of the turbulent centerline wake.

The dynamics of low-density buoyancy-driven surface currents, also referred to as "plumes", and their interaction with ambient near-surface stratification, as well as an internal wave soliton are also investigated. Buoyancy-driven surface currents, such as those within propagating river or rainformed plumes, are an important component of the oceanic environment. These currents contribute to water mass exchange by horizontal advection and enhanced vertical mixing. Such buoyancy-driven currents propagate in the nearsurface layer resembling a classical gravity current (Baines, 2001; Benjamin, 1968; Simpson, 1987). The currents are driven by the density difference between the gravity current front and the ambient water into which it propagates, and they are generally associated with enhanced mixing.

These buoyancy-driven currents may also interact in a resonant way with the continuously stratified ambient environment. This can lead to a fragmentation of the density current, as seen in laboratory experiments by Simpson (1987) and open-ocean observations by Soloviev and Lukas (1997). A buoyancy-driven current propagating into a two-layer stratified ambient environment in the coastal ocean may result in a resonant generation of an upstream undular bore (Grimshaw and Yi, 1991; Nash and Moum, 2005; White and Helfrich, 2012). In the presence of wind stress, an asymmetry may develop in the sea surface signature of low-density plumes (Fujimura et al., 2011). Here, we investigate the propagation of a low-density buoyancy-driven current in the presence of a continuous upper ocean stratification.

As part of this work, the use of CFD to study the magnetic signatures of oceanic fine-scale processes is explored. Ocean surface and internal waves have been reported to lead to a measurable signal in the ocean electromagnetic field (Crews and Futterman, 1962; Beal and Weaver, 1970). These magnetic field perturbations arise by induction, through the motion of the conducting sea water through the earth's magnetic field. The magnetic fields induced by oceanographic processes may be an important source of noise in aeromagnetic surveys (Chave, 1986). We have been able to simulate electromagnetic signatures of internal waves, buoyancy-driven currents associated with low-density nearsurface plumes, and ship wakes in the ocean magnetic field. Based on our results, we suggest that combining SAR imagery with data from ocean magnetometers has the potential to become a valuable additional maritime sensing method.

The paper is structured as follows. Section 2 describes the hydrodynamic and electromagnetic model, as well as the model setup and parameters. It briefly discusses the usage of the radar imaging model. In Sect. 3, the results from our numerical simulations are presented. Summary and discussion conclude the paper in Sect. 4.

\section{Methods}

CFD models, by default, are non-hydrostatic and often threedimensional and high-resolution numerical models that are routinely applied to engineering problems. Here, the CFD code ANSYS Fluent is used to study the dynamics of upper ocean fine-scale processes, which are inherently threedimensional and non-hydrostatic. The model uses a control volume approach to solve conservation equations for mass and momentum. The following sections describe both hydrodynamic (more details can be found in Matt et al., 2011) and electromagnetic model, as well as the model setup and parameters. The detailed description of the M4S radar imaging model used in this study is beyond the scope of this paper, and we provide only a brief overview of its usage.

\subsection{Hydrodynamic model}

Despite recent advances in computing power, it is still computationally prohibitive to numerically solve the fully threedimensional Navier-Stokes equations for turbulent processes at all scales and for realistic domain sizes. In the so-called large eddy simulation (LES) turbulence model, the assumption is that most of the energy is contained in the large eddies and overturns in the flow, and hence the model aims to explicitly resolve the scales of these large eddies, while the subgrid scales are modeled. The governing equations for LES are obtained by filtering the time-dependent Navier-Stokes equations. The process filters out eddies with scales smaller than the filter width or grid spacing, and the resulting equations govern the dynamics of the large eddies (Sagaut, 1998).

The model solves the filtered Navier-Stokes equations (e.g., see Lesieur, 2008). The subgrid-scale stresses, defined, in Cartesian tensor notation, by

$\tau_{i j} \equiv \rho \overline{u_{i} u_{j}}-\rho \bar{u}_{i} \bar{u}_{j}$,

which are resulting from the filtering operation, need to be modeled. Here, $\rho$ is the fluid density, $u$ is the fluid velocity $(i=1,2,3)$, and the overbar denotes a filtered variable. 
Using the Boussinesq hypothesis (Hinze, 1975), the subgrid-scale stresses are computed from

$\tau_{i j}-\frac{1}{3} \tau_{k k} \delta_{i j}=-2 \mu_{\mathrm{t}} \bar{S}_{i j}$,

where $\mu_{\mathrm{t}}$ is the subgrid-scale turbulent viscosity and $\delta_{i j}$ is the Kronecker delta. $\tau_{k k}$ is the isotropic part of the subgridscale stresses, which is not modeled but added to the term containing the static pressure in the filtered Navier-Stokes equations. The rate-of-strain tensor for the resolved scales is

$\bar{S}_{i j}=\frac{1}{2}\left(\frac{\partial \bar{u}_{i}}{\partial x_{j}}+\frac{\partial \bar{u}_{j}}{\partial x_{i}}\right)$.

To calculate the turbulent viscosity $\mu_{\mathrm{t}}$, we apply the socalled Wall-Adapting Local Eddy-Viscosity (WALE) model (Nicoud and Ducros, 1999) for the simulation of the ship wake and low-density buoyant plume in the near-surface layer of the ocean. The WALE model improves the LES performance near a solid boundary. For the simulation of an internal wave in the thermocline, the traditional SmagorinskyLilly model is used (Smagorinsky, 1963).

The subgrid-scale terms in the filtered energy equation, namely the subgrid enthalpy flux, are approximated as

$\rho\left(\overline{u_{i} h_{\mathrm{s}}}-\bar{u}_{i} \bar{h}_{\mathrm{s}}\right)=-\frac{\mu_{\mathrm{t}} C_{p}}{\sigma_{\mathrm{t}}} \frac{\partial \bar{T}}{\partial x_{j}}$,

where $T$ is the temperature, $\sigma_{\mathrm{t}}$ is the subgrid-scale turbulent Prandtl number, $\sigma_{\mathrm{t}}=0.85, C_{p}$ is the specific heat, and $h_{\mathrm{s}}$ is the sensible enthalpy.

Finally, the subgrid-scale turbulent flux $q_{j}$ of a scalar $\Phi$ is calculated from

$q_{j}=-\frac{\mu_{\mathrm{t}}}{\sigma_{\mathrm{t}}} \frac{\partial \Phi}{\partial x_{j}}$.

\subsection{Electromagnetic model}

The equations governing a magnetic field are the Maxwell equations (Crews and Futterman, 1962). In vector notation, we have

$\nabla \times \frac{\boldsymbol{B}}{\mu_{\mathrm{m}}}=\boldsymbol{J}$,

$\frac{1}{\sigma} \boldsymbol{J}=\boldsymbol{E}+\boldsymbol{v} \times \boldsymbol{B}$,

$\frac{\partial \boldsymbol{B}}{\partial t}=-\nabla \times \boldsymbol{E}$.

Here, $\mu_{\mathrm{m}}$ is the magnetic permeability and $\sigma$ is the electrical conductivity of the fluid. $\boldsymbol{B}$ is the magnetic field, $\boldsymbol{E}$ denotes the electric field, $\boldsymbol{J}$ is the electric current density, and $\boldsymbol{v}$ is the fluid velocity.
Combining Ampère's law (6) and Ohm's law (7), we get

$\boldsymbol{E}=-\boldsymbol{v} \times \boldsymbol{B}+\frac{1}{\mu_{\mathrm{m}} \sigma} \nabla \times \boldsymbol{B}$,

and the Maxwell-Faraday Eq. (8) becomes

$$
\frac{\partial \boldsymbol{B}}{\partial t}=\nabla \times(\boldsymbol{v} \times \boldsymbol{B})-\nabla \times\left(\frac{1}{\mu_{\mathrm{m}} \sigma} \nabla \times \boldsymbol{B}\right) .
$$

Equation (10) then transforms as follows:

$$
\begin{aligned}
\frac{\partial \boldsymbol{B}}{\partial t} & +(\boldsymbol{v} \cdot \nabla) \boldsymbol{B}=(\boldsymbol{B} \cdot \nabla) \boldsymbol{v}+\frac{1}{\mu_{\mathrm{m}} \sigma} \nabla^{2} \boldsymbol{B}+\frac{1}{\sigma}(\nabla \sigma) \\
& \times \boldsymbol{E}+\frac{1}{\sigma} \nabla \sigma \times(\boldsymbol{v} \times \boldsymbol{B}) .
\end{aligned}
$$

In ANSYS Fluent, the induction Eq. (11) is implemented for constant conductivity $\sigma$. The last two terms on the right-hand side of Eq. (11) are neglected. These terms are important in situations where one needs to account for variable conductivity, such as across the air-sea interface. However, since in this paper, we are interested in the induced magnetic fields in the water only, we use the equation for constant conductivity provided within the Fluent software.

The magnetic field can generally be decomposed into the externally imposed field $\boldsymbol{B}_{0}$ and the induced field $\boldsymbol{b}$, which is due to fluid motion. Only the induced field needs to be solved and, in a conducting medium, the model solves the following equations:

$$
\begin{aligned}
\frac{\partial \boldsymbol{b}}{\partial t}+(\boldsymbol{v} \cdot \nabla) \boldsymbol{b} & =\left(\left(\boldsymbol{B}_{0}+\boldsymbol{b}\right) \cdot \nabla\right) \boldsymbol{v}+\frac{1}{\mu_{\mathrm{m}} \sigma} \nabla^{2} \boldsymbol{b} \\
& -(\boldsymbol{v} \cdot \nabla) \boldsymbol{B}_{0},
\end{aligned}
$$

$\boldsymbol{J}=\frac{1}{\mu_{\mathrm{m}}} \nabla \times\left(\boldsymbol{B}_{0}+\boldsymbol{b}\right)$

\subsection{Radar imaging model}

We combine the results from the CFD model with the radar imaging model M4S (Romeiser, 2008). M4S is a suite of numerical models for radar signature simulations. It consists of a wave-current interaction module based on weak interaction theory in the relaxation-time approach, a composite surface radar backscattering module based on Bragg scattering theory, and additional modules for specific radar data product simulations that have not been used for this study. The two main modules of M4S were described in the papers by Romeiser and Alpers (1997) and Romeiser et al. (1997), respectively. M4S has been used for a variety of studies on radar signatures of oceanic and atmospheric features, including studies on signatures of Kelvin arms of ship wakes (Hennings et al., 1999), oceanic fronts (Ufermann and Romeiser, 1999), and oceanic internal waves (Brandt et al., 

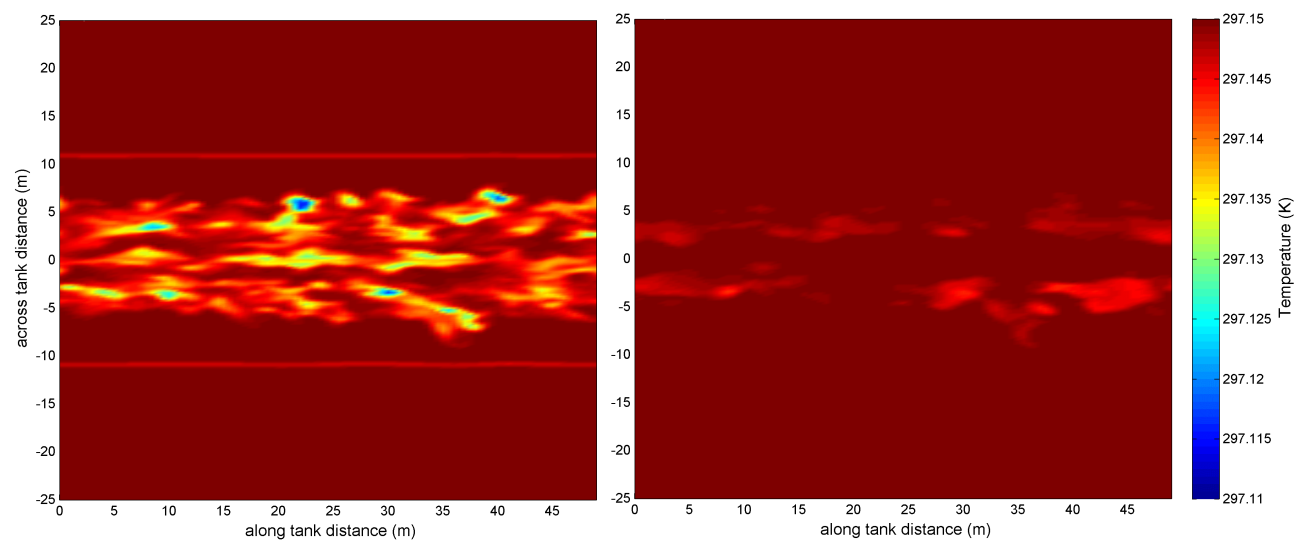

Figure 1. Top view of temperature field (in kelvin, at time $t=575 \mathrm{~s}$ ) for ship wake model in the presence of an ambient stratification illustrates the upwelling of colder subsurface water by the circulation in the ship wake. Left: at depth $0.5 \mathrm{~m}$; right: at depth $5 \mathrm{~mm}$. The temperature difference at the surface is small in our case, but is expected to be more pronounced in the case of a larger near-surface temperature gradient and stronger wake circulation.
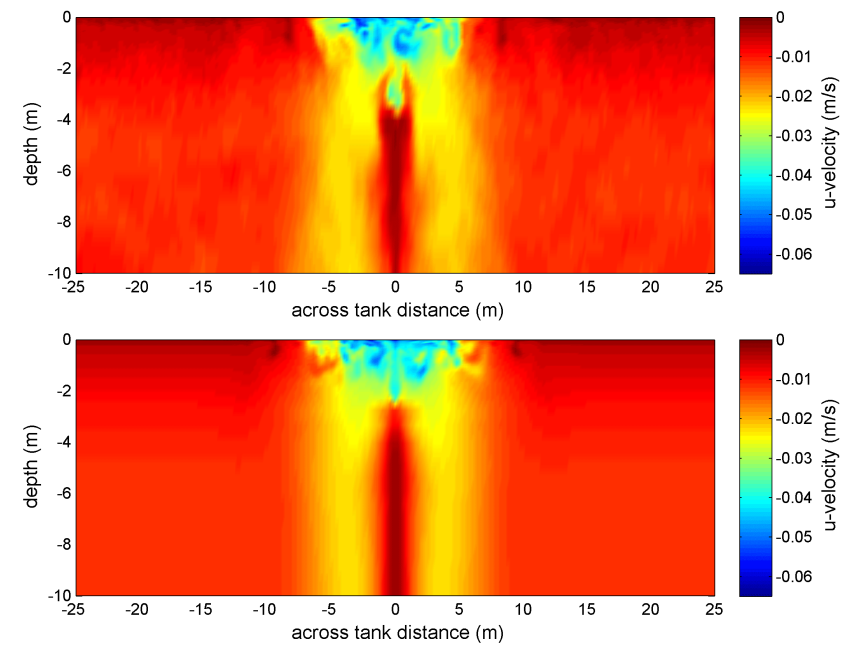

Figure 2. Side view of $x$ velocity (at time $t=575 \mathrm{~s}$ ) for ship wake model without (top panel) and with (bottom panel) ambient stratification. The stratification appears to affect the spreading of the wake at the surface and to suppress background turbulent fluctuations in the model. Also note that the model shows a region of low velocity at mid-depth, centered below the surface circulation.

1999). It has been found to reproduce observed radar signatures well qualitatively, but to underestimate observed modulation depths quantitatively in many cases, which has been attributed to a neglect of nonlinear effects such as wave breaking. In most cases, the underestimation of radar signatures could be compensated by reducing the relaxation rate in the wave-current interaction module of M4S (see Brandt et al., 1999, as an example).

Within the framework of this work, it would be unrealistic to strive for perfect agreement between simulated and observed radar signatures because a lot of simplifications have been made in our hydrodynamic modeling. For instance, the hydrodynamic model does not have a free surface, and thus it will not allow the development of a surface wave field. The surface velocities from the hydrodynamic model represent the velocities associated with surface currents due to the underlying hydrodynamic features. M4S is based on weak hydrodynamic interaction theory and allows for the computation of the wave height spectral density for a given set of wavenumbers and directions at arbitrary positions in a given current field. Thus, the algorithm is designed to estimate the local modulation of the wave spectrum due to surface currents.

Despite the limitations due to model assumptions, we can use M4S to demonstrate a qualitative agreement between theoretical radar signatures of the simulated current features and observed signatures in SAR images, indicating that some characteristic properties of our simulated current fields seem to be realistic.

The approach of combining results from CFD with a radar imaging model has previously been introduced by Fujimura et al. (2010). The surface velocity fields from the hydrodynamic model are used as input for the radar model. The settings for the radar imaging model here are $9.65 \mathrm{GHz}(\mathrm{X}-$ band), $\mathrm{HH}$ polarization, $35^{\circ}$ incidence angle, and $180^{\circ}$ look direction. This corresponds to a look direction along the $x$ direction of the CFD model domains. We explored the use of different look directions in the M4S settings and found the choice did not significantly affect our results. Here, we ignore the effect of wind stress on the hydrodynamics; however, we set a wind speed of $4 \mathrm{~m} \mathrm{~s}^{-1}$ along the $x$ axis in the M4S model.

\subsection{CFD model setup and parameters}

The model used a pressure-based solver, where pressurevelocity coupling was achieved based on a predictorcorrector approach, namely the Pressure-Implicit with Splitting of Operators (PISO) scheme (Issa, 1982). For spatial 
Table 1. Material properties.

\begin{tabular}{|c|c|c|c|c|c|c|}
\hline Experiment & $\begin{array}{r}\text { Molecular } \\
\text { dynamic } \\
\text { viscosity } \mu \\
\mathrm{kg} \mathrm{m}^{-1} \mathrm{~s}^{-1}\end{array}$ & $\begin{array}{r}\text { Thermal } \\
\text { conductivity } \\
\kappa \\
\mathrm{W} \mathrm{m}^{-1} \mathrm{~K}^{-1}\end{array}$ & $\begin{array}{r}\text { Magnetic } \\
\text { permeability } \\
\mu_{\mathrm{m}} \\
\mathrm{H} \mathrm{m}^{-1}\end{array}$ & $\begin{array}{r}\text { Electrical } \\
\text { conductivity } \\
\text { Electrical } \sigma \\
\mathrm{S} \mathrm{m}^{-1}\end{array}$ & $\begin{array}{r}\text { Specific } \\
\text { heat } c_{p} \\
\mathrm{~J} \mathrm{~kg}^{-1} \mathrm{~K}^{-1}\end{array}$ & $\begin{array}{r}\text { Density } \\
\rho \\
\mathrm{kg} \mathrm{m}^{-3}\end{array}$ \\
\hline Ship wake, without stratification & $1.08 \times 10^{-3}$ & 0.596 & - & - & 3993 & 998.2 \\
\hline Ship wake with stratification; Plume; Internal Wave & $1.08 \times 10^{-3}$ & 0.596 & $1.257 \times 10^{-6}$ & 4.788 & 3993 & 1024.75 \\
\hline
\end{tabular}

Table 2. Grid dimensions and time steps for ship wake, low-density plume and internal wave simulations. The vertical resolution for the ship wake and plume model is highest near the top of the domain and decreases towards the bottom. For the internal wave model, the highest vertical resolution is in the thermocline.

\begin{tabular}{lrrrrrrr}
\hline Experiment & Length $x, \mathrm{~m}$ & Width $y, \mathrm{~m}$ & Height $z, \mathrm{~m}$ & $\Delta x, \mathrm{~m}$ & $\Delta y, \mathrm{~m}$ & $\Delta z, \mathrm{~m}$ & Time step $\Delta t, \mathrm{~s}$ \\
\hline Ship wake & 50 & 50 & 10 & 0.5 & 0.2 & 0.001 (top cell) & 0.025 \\
Plume & 500 & 40 & 40 & 0.5 & 1 & 0.01 (top cell) & 0.5 \\
Internal wave & 2000 & 1000 & 250 & 10 & 10 & $1-2$ & 5 \\
\hline
\end{tabular}

discretization, a staggered-grid scheme was used for pressure (Patankar, 1980) and second-order accuracy schemes for the momentum, energy and induction equations. For the time-stepping, a second-order accuracy, implicit scheme was used. The material properties in the domain were specified as those of pure water or sea water (Table 1) using the Boussinesq model, which treats density as a constant value in all solved equations, except for the buoyancy term in the momentum equation. This model uses the linearized equation of state for density. The operating pressure was set to atmospheric pressure of $101325 \mathrm{~Pa}$. Background stratification for the ship wake model changed linearly from $T=296.65 \mathrm{~K}$ to $T=297.15 \mathrm{~K}$ in the depth range from -6 to $-1 \mathrm{~m}$ and from $T=294.15 \mathrm{~K}$ to $T=297.15 \mathrm{~K}$ in the depth range from -16 to $-6 \mathrm{~m}$ for the surface buoyancy current model. The unstratified ship wake model had a constant initial temperature of $293.15 \mathrm{~K}$. The stratification for the internal wave model was chosen to resemble a typical January stratification on the south-east Florida shelf and ranged from $T=283.15 \mathrm{~K}$ to $T=293.15 \mathrm{~K}$ changing linearly in the depth range from -200 to $-100 \mathrm{~m}$. The operating temperature was set to $297.15 \mathrm{~K}$ for the ship wake $(293.15 \mathrm{~K}$ for the unstratified case), $293.15 \mathrm{~K}$ for the plume simulations, and $288.15 \mathrm{~K}$ for the internal wave simulation. The grid dimensions and time steps for ship wake, low-density plume and internal wave simulations are given in Table 2.

For the simulations using the electromagnetic model, the external magnetic field was set to that of the earth's magnetic field close to our location in South Florida, with the components $B_{0_{x}}=-2.7 \times 10^{-6} \mathrm{~T}, B_{0_{y}}=2.52 \times 10^{-5} \mathrm{~T}$ and $B_{0 z}=-3.7 \times 10^{-5} \mathrm{~T}$. Following validation of the steadystate Fluent magneto-hydrodynamics (MHD) solver with a known electromagnetic model used to simulate ocean magnetic fields induced by internal waves (Soloviev et al., 2013), the solution procedure used in this paper was as follows: the hydrodynamic solution was calculated first, and the induced magnetic field was then calculated from the existing hydrodynamic fields, holding the hydrodynamic solution constant and using the steady-state MHD solver. Convergence of the model solution was generally achieved within 1000-2000 iterations.

The boundary conditions for the hydrodynamic model, for both ship wake and near-surface low-density plume, were set to a translationally periodic condition along the wake and in the direction of the buoyancy current propagation and to zero shear stress (equivalent of free-slip condition) and no heat flux at all other sides. The boundary conditions for the electromagnetic model of the ship wake were conducting at the bottom, and insulating at the top and non-periodic sides. The same boundary conditions were applied for the low-density plume, bottom conducting, the top and the non-periodic sides insulating. Here, the use of periodic boundary conditions allows us to investigate the time evolution of the flow without constraint from the along-flow boundaries, especially in the case of the turbulent centerline wake.

The boundary conditions for the internal wave simulations for the hydrodynamic model were a zero shear stress wall at the eastern boundary and outflow at the western boundary (along the direction of wave propagation), as well as a zero shear stress wall at the top and a no-slip bottom. The northsouth sides (across the direction of wave propagation) were set to velocity inlet conditions with the temperature set to a prescribed initial temperature profile. This was done to account for any mass lost at the outflow boundary. The inflow coming through these sides is negligible in our simulations. All other boundaries were set to zero heat flux. The boundary conditions on the electromagnetic model were set to conducting at the bottom, to zero flux at the velocity inlets and insulating at the top and at the eastern wall.

The simulations were run in parallel on eight processors on Dell Precision T7400 work stations with two Quad-Core Intel Xeon processors and 64 bit Windows operating system. 

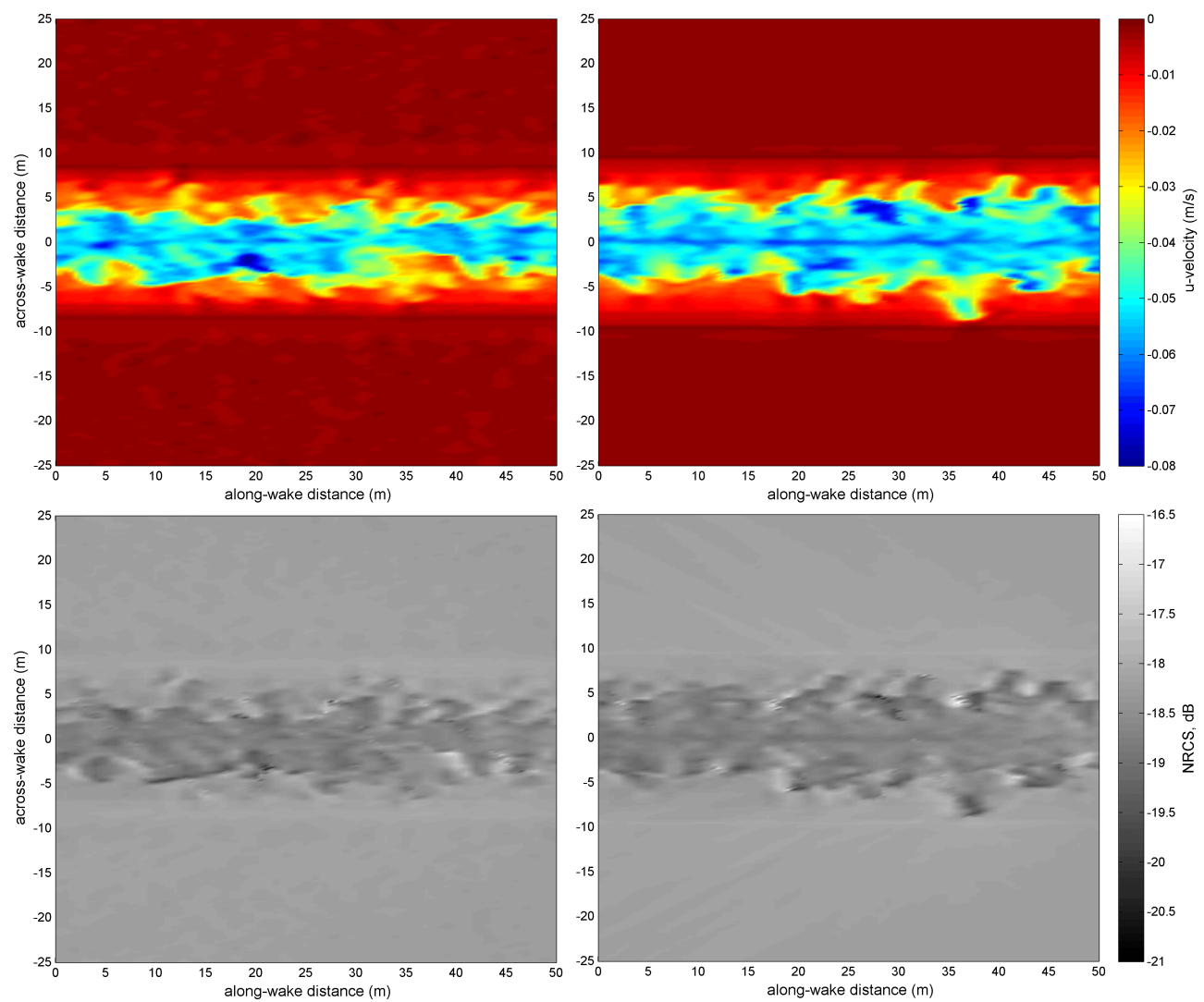

Figure 3. Ship wake signature at the surface in the velocity field (top, in $\mathrm{m} \mathrm{s}^{-1}$ ) and radar backscatter intensity map (bottom; as normalized radar cross section (NRCS), $\mathrm{dB}$ values here should be taken as a relative range) for the case without ambient stratification (left) and with ambient stratification (right). Taken at time $t=575 \mathrm{~s}$.

\section{Results}

\subsection{Ship wakes}

In this paper, our interest is in the turbulent centerline wake. The ship wake model used in this study has previously been described by Fujimura et al. (2011). This model of the far wake of a ship is initialized with velocity fields from a near wake simulation of flow around a surface ship hull with modeled propeller wakes (Rhee and Kim, 2008). The periodic boundary conditions along the wake allow the investigation of the time evolution of the wake, simulating a decaying wake after the passage of a vessel.

Here, we investigate the effect of a thermal stratification in the oceanic near-surface layer on the wake hydrodynamics and surface signature. Our simulations suggest that cold subsurface water may be brought to the surface through the circulation in the ship wake (Fig. 1). Since the temperature difference in the stratification set in our model is only $0.5 \mathrm{~K}$ across the top $7 \mathrm{~m}$ of the water column, the signal we find in our results is very weak. However, the temperature anomaly seen at the surface can be expected to be more pronounced in the case of a larger near-surface temperature gradient and

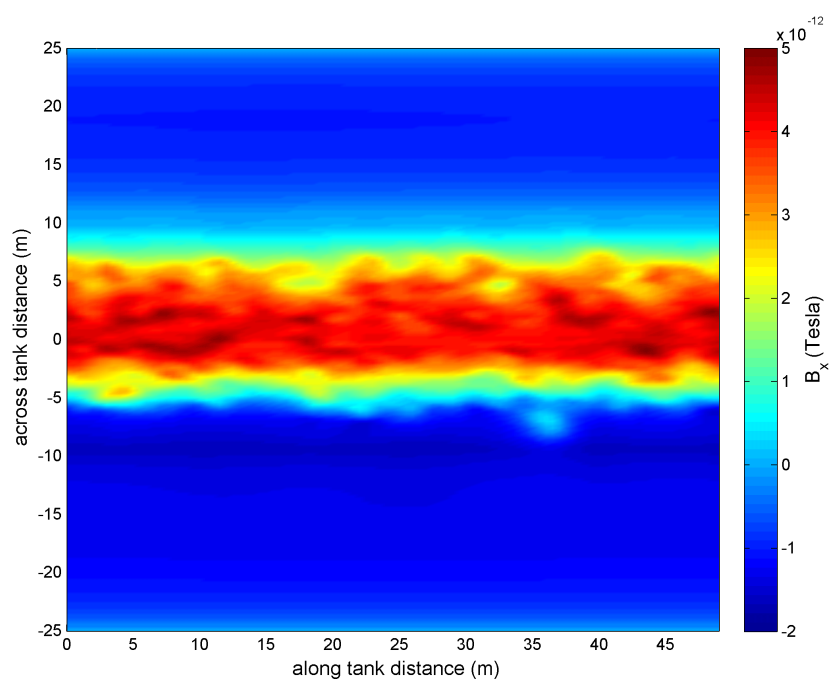

Figure 4. Top view of ship wake signature in the induced magnetic field for the case with ambient stratification at time $t=575$. The $x$ component of the induced magnetic field is shown at $1 \mathrm{~m}$ below the surface. 


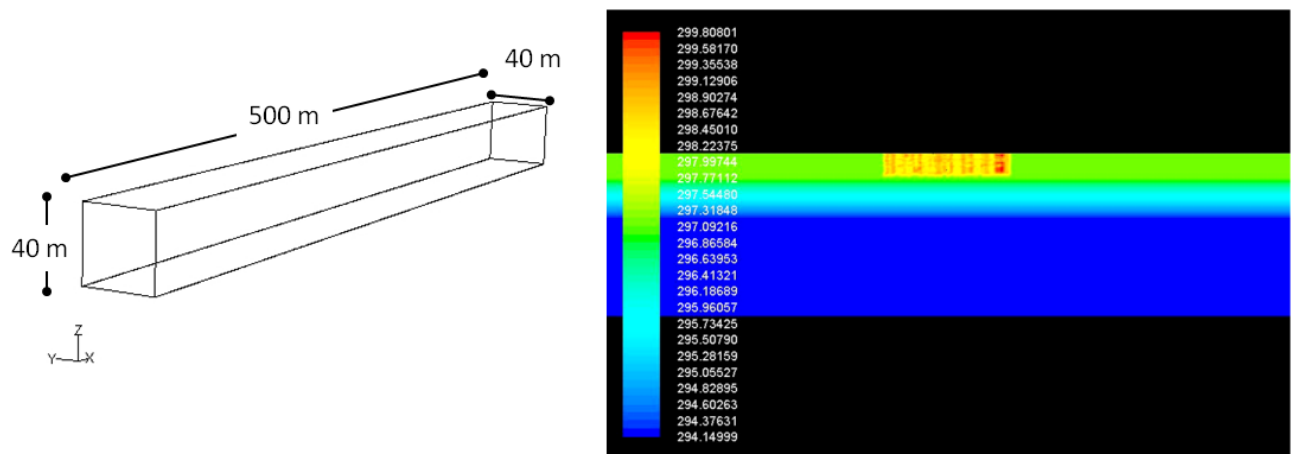

Figure 5. Model domain (left) and side view of initial condition in the temperature field (right; at $t=0 \mathrm{~s}$, in kelvin) for the low-density plume simulation. Model horizontal resolution is $0.5 \mathrm{~m}$ in $x$ by $1 \mathrm{~m}$ in $y$ and vertical resolution is $1 \mathrm{~cm}$ at the top (with decreasing vertical resolution towards the bottom).
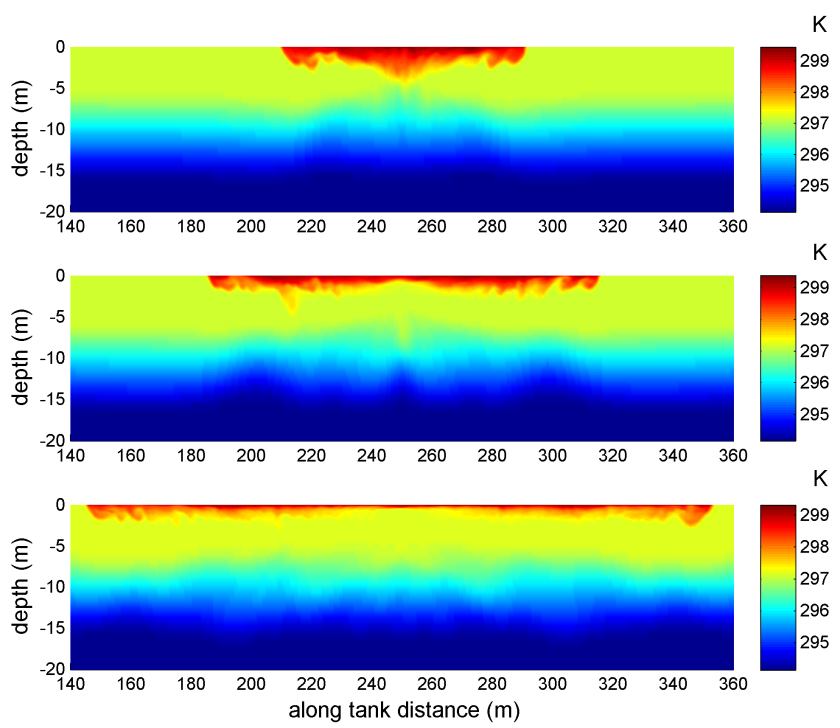

Figure 6. Close-up of a side view of temperature (in kelvin) illustrates the propagation of the low-density buoyancy-driven current in the near-surface layer. The propagating flow has the form of a classical gravity current with a leading head and trailing tail region. Internal waves can be seen in the thermocline. Time evolution is from top to bottom. Top: after time $300 \mathrm{~s}$, middle: after $1500 \mathrm{~s}$, bottom: after $2000 \mathrm{~s}$.

stronger wake circulation than seen in our model. Qualitatively, our result is consistent with in situ observations of a ship wake signature in the temperature field in the Straits of Florida presented in a companion paper (Soloviev et al., 2012). Such a sea surface signature in the temperature field may also be detected by high-resolution infrared cameras.

Stratification also appears to have an effect on the surface spreading of the wake, as well as on subsurface small-scale turbulent fluctuations (Fig. 2). In the presence of stratified ambient waters, the wake circulation in the shallower layers of the water column appears more compact, with less of a downward extent. The sea surface signature of the wake can
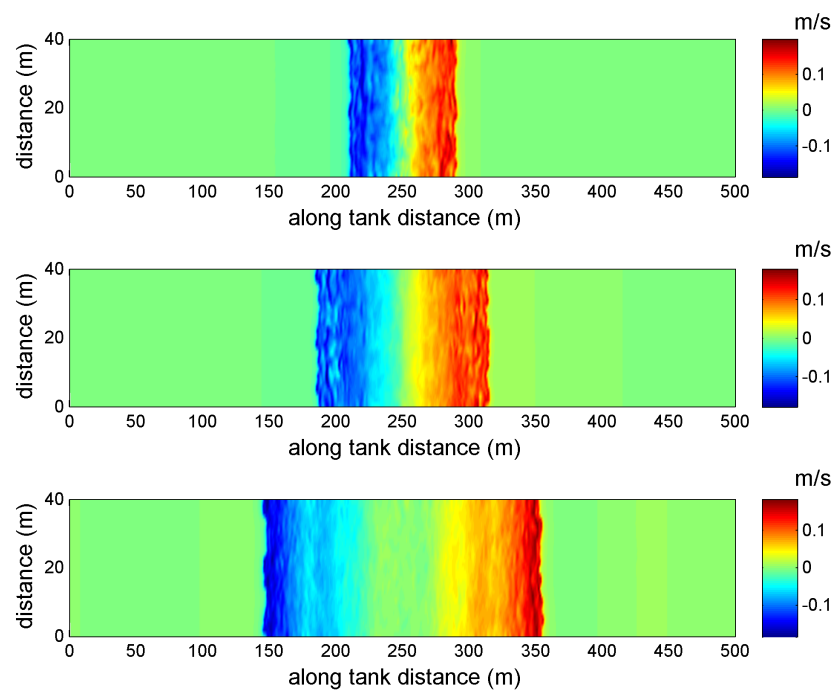

Figure 7. Top view of the along-tank $(x)$ velocity (in $\mathrm{m} \mathrm{s}^{-1}$ ) showing the evolution of the density current front and the fragmentation (surface "lines") that occurs due to the interaction of the density current with the ambient stratification. Top: after time $300 \mathrm{~s}$, middle: after 1500 s, bottom: after $2000 \mathrm{~s}$.

be seen in the velocity field as well as in the radar backscatter intensity map, which is a proxy for a SAR image. In the case of a near-surface thermal stratification, the wake surface signature appears wider and more pronounced than is the case without stratification (Fig. 3). Note that here we are considering the turbulent centerline wake only; however, M4S has previously been used to successfully simulate the Kelvin arms of a ship wake (Hennings et al., 1999). The simulated radar images of the ship wake exhibit characteristics that are consistent with those seen in real SAR images of the wake of a small vessel (Toporkov et al., 2011), such as a brightdark-bright banding pattern across the wake. Furthermore, the relative range of normalized radar cross section (NRCS) across the wake, in $\mathrm{dB}$, is comparable to the SAR intensity 

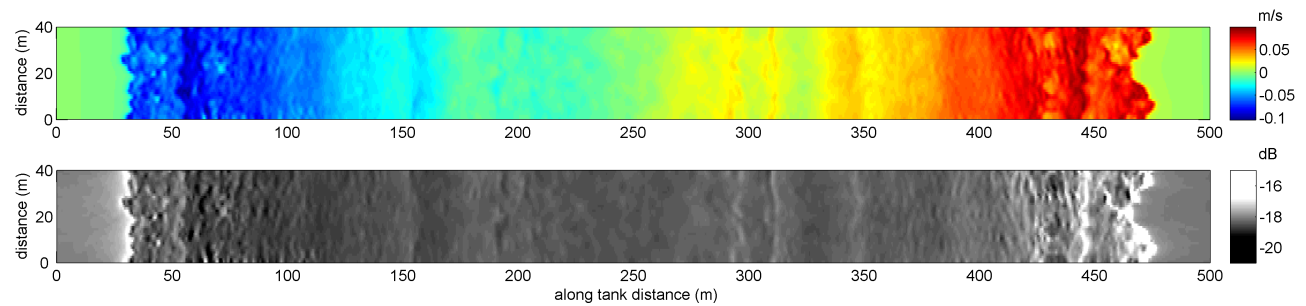

Figure 8. Surface signature of low-density plume in the velocity field (top, in $\mathrm{m} \mathrm{s}^{-1}$ ) and radar backscatter intensity map (bottom; NRCS, $\mathrm{dB}$ values here should be taken as relative range). The $x$ axis shows along-tank distance; $y$ axis is across-tank distance (in $\mathrm{m}$ ). Taken at time $t=2850 \mathrm{~s}$.

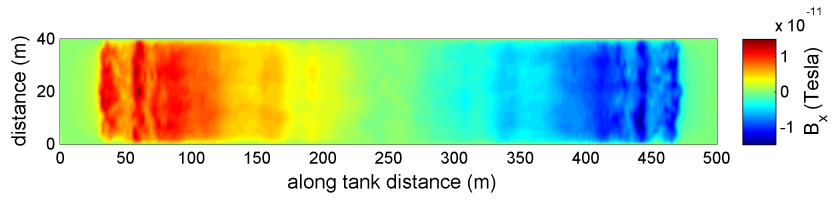

Figure 9. Signature of the low-density buoyancy-driven current in the induced magnetic field ( $x$ component) at $1 \mathrm{~m}$ below the surface at time $t=2850 \mathrm{~s}$.

range found by Toporkov et al. (2011), though they report velocities in the wake that exceed the velocities seen in our model.

Combining the ship wake hydrodynamic model with the electromagnetic model within the CFD framework allows the investigation of the ship wake signature in the ocean magnetic field. The induced field reaches values of the order of $10^{-12} \mathrm{~T}$, which is less than what is possible to detect with modern magnetometers (Fig. 4). However, the idealized and scaled ship model for our ship wake model is relatively small ( $\sim 6 \mathrm{~m}$ length), and we expect to see a larger signal for a larger vessel with more intense wake circulation and thus higher velocities. Furthermore, in our simulations, we use as external field strength the value for the earth's magnetic field close to our location in South Florida. At higher latitudes, the induced magnetic field is expected to be larger due to an increase in the strength of the geomagnetic field near the poles.

Note that our results suggest that, in an oceanic setting, the ship's wake may be detected in the magnetic field for tens of minutes after the ship has passed through the observational domain. Combining data from magnetometers with SAR imagery of ship wakes may add a valuable new dimension to ship surveillance and detection.

\subsection{Near-surface buoyancy-driven currents}

The setup and initial conditions for the simulation of nearsurface buoyancy-driven currents associated with a lowdensity plume are shown in Fig. 5. The model is initialized with a density anomaly in the near-surface layer, simulating a rain-formed plume. Here, temperature is used to generate the density anomaly. This parcel of low-density water
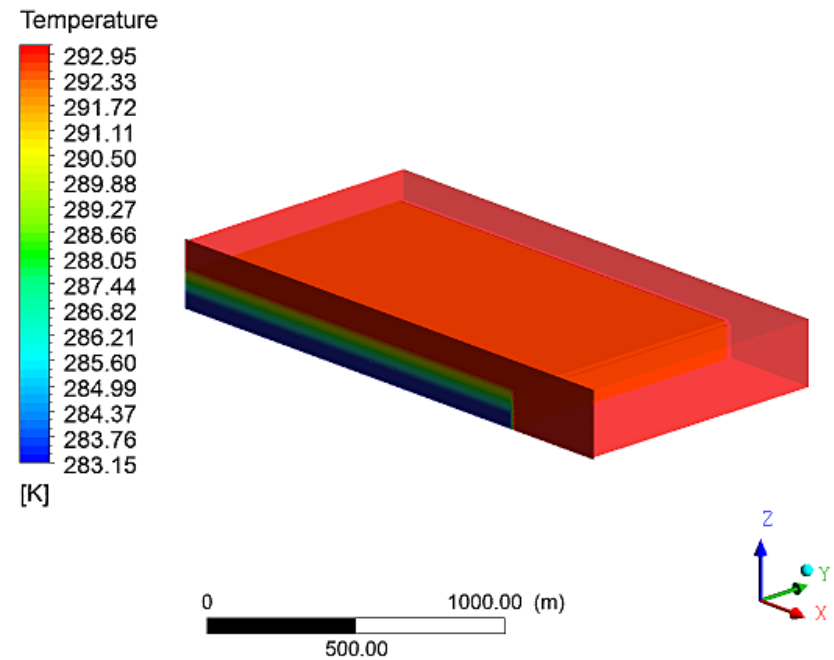

Figure 10. Initial condition for internal wave simulation shown in the model temperature field (in kelvin). Domain length $(x)$ is $2 \mathrm{~km}$; domain width $(y)$ is $1 \mathrm{~km}$; domain depth $(z)$ is $250 \mathrm{~m}$. Model horizontal resolution is $10 \mathrm{~m}$ and vertical resolution $2 \mathrm{~m}$, with increased vertical resolution in the thermocline.

then propagates in the upper layer of the water column as a buoyancy-driven current. The flow exhibits features of a classic gravity current, including the leading gravity current head and a trailing tail region, where Kelvin-Helmholtz overturns are apparent, which are known to contribute to mixing (Fig. 6). Such density-driven currents are a wellknown phenomenon in environmental fluid dynamics and have been extensively studied theoretically and in the laboratory (Baines, 2001; Benjamin, 1968; Simpson, 1987). As a near-surface low-density plume propagates into the stratified environment, internal waves can be excited in the thermocline. This can lead to a resonant interaction between the internal waves and the near-surface current, which results in a fragmentation of the buoyancy-driven current at the surface as observed in laboratory experiments (Simpson, 1987) and in the open ocean (Soloviev and Lukas, 1997). The fragmentation can become apparent as a banding pattern on the sea surface, as seen in the velocity field in our simulations (Fig. 7). This pattern of alternating bands can also be seen 

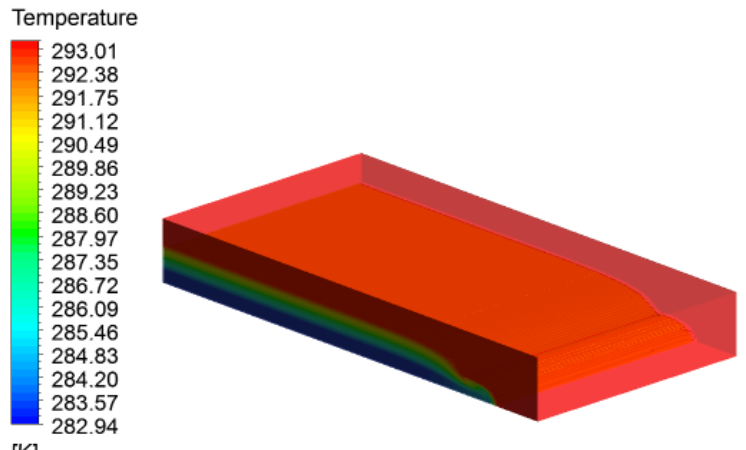

[K]

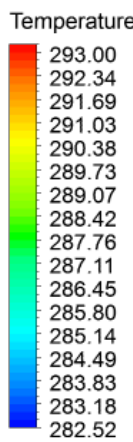

$[\mathrm{K}]$

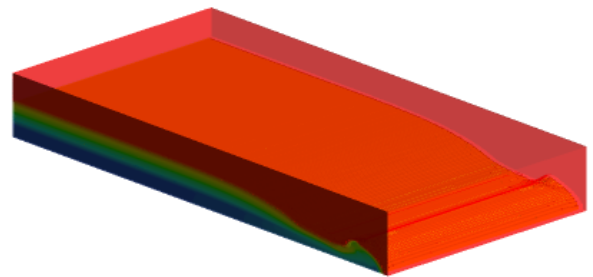

Temperature

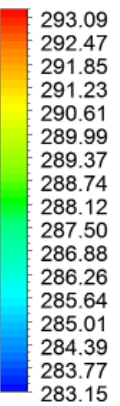

$[\mathrm{K}]$
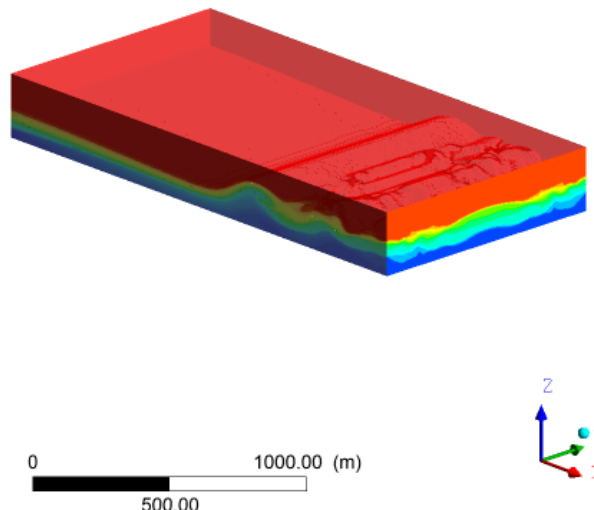

Figure 11. Evolution of internal wave simulation in the model temperature field (in kelvin). Time evolution is from top to bottom. Top: after time $200 \mathrm{~s}$, middle: after $400 \mathrm{~s}$, bottom: after $1800 \mathrm{~s}$.

in the sea surface signature in the simulated radar image (Fig. 8). Under certain conditions, river plumes are known to generate large amplitude internal waves at their leading edge (Nash and Moum, 2005), which, however, may in part be due to a different mechanism of resonant interaction (White and Helfrich, 2012). These can exhibit a signature on the sea surface in SAR similar to that seen in our results.

Our results are consistent with observations of freshwater plumes in the western equatorial Pacific (Soloviev and
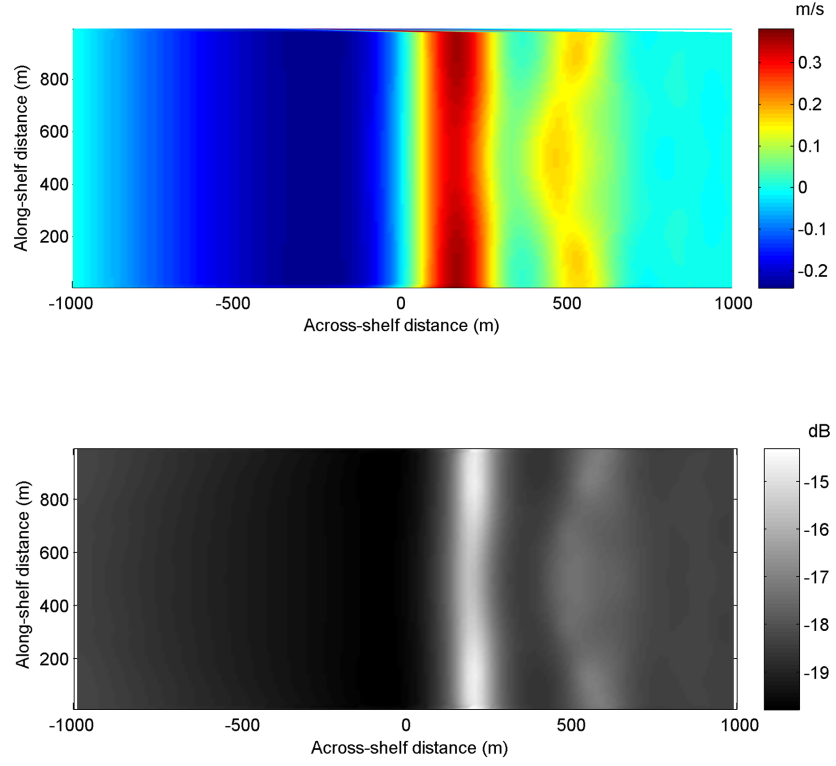

Figure 12. Internal wave signature in velocity field (top) at $1 \mathrm{~cm}$ depth and radar backscatter intensity map (bottom; NRCS, dB values here should be taken as a relative range). Taken at time $t=$ $2000 \mathrm{~s}$.

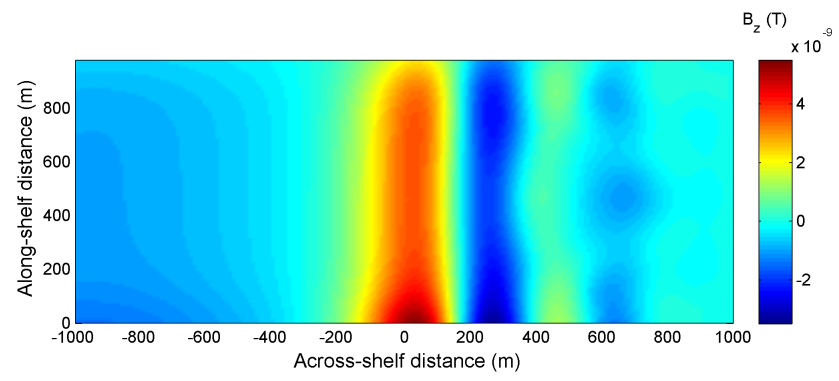

Figure 13. Internal wave signature in the induced magnetic field ( $z$ component) at $1 \mathrm{~m}$ depth, at time $t=2000 \mathrm{~s}$.

Lukas, 1997). Following Soloviev and Lukas (2006), to generate internal waves the Froude number $\mathrm{Fr}$ should satisfy

$F r=\frac{\sqrt{g^{\prime} h}}{N H}<1 / \pi$

and

$\frac{h}{H}<0.2$

Here, $g^{\prime}=g \frac{\Delta \rho}{\rho_{0}}$ is the reduced gravity, $N^{2}=-\frac{g}{\rho_{0}} \frac{\partial \rho}{\partial z}$ is the Brunt-Väisällä frequency, $h$ is the depth of the gravity current, and $H$ is the total water depth. Conditions (14) and (15) are satisfied in the numerical experiments presented here.

When investigating the magnetic signature associated with the low-density buoyancy-driven current, it is found that the magnetic signal follows the propagating density current front and reaches magnitudes of the order of $10^{-11} \mathrm{~T}$ (Fig. 9). A 

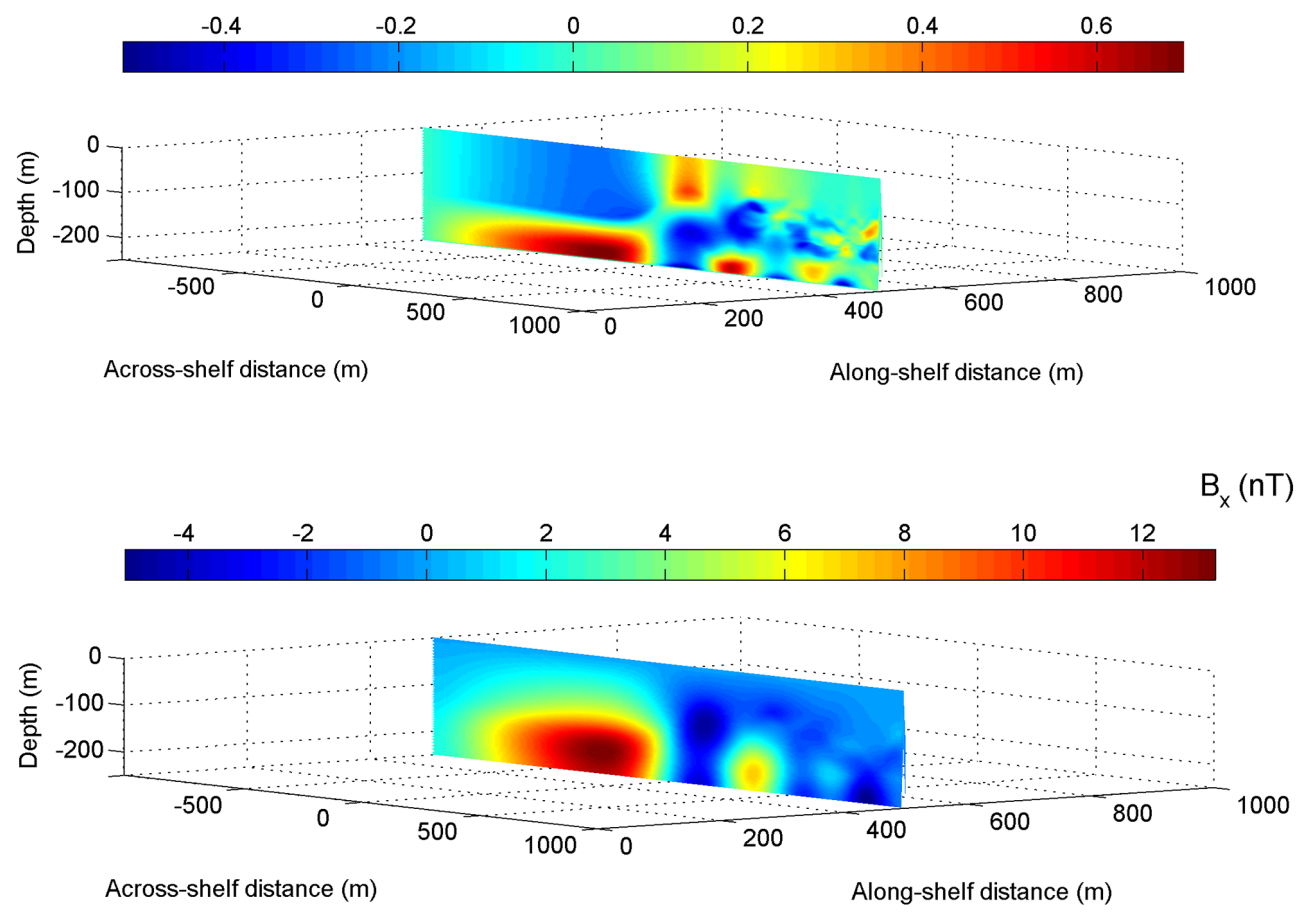

Figure 14. Top: side view of the velocity component in direction of wave propagation (in $\mathrm{m} \mathrm{s}^{-1}$ ) at time $t=2000 \mathrm{~s}$. Bottom: side view of the $x$ component of the induced magnetic field (in nT) at time $t=2000 \mathrm{~s}$.

propagating rain-formed or river plume is therefore expected to produce a measurable magnetic signature, especially for cases with large freshwater inflow and strong density contrast.

\subsection{Internal waves}

The model setup for the simulation of an internal wave soliton and the ambient stratification (Fig. 10) were chosen to resemble the environment observed in the Straits of Florida by Soloviev et al. (2012) on 16 January 2011. The wave is generated via a density anomaly that initially propagates as gravity current but after reflection from the wall boundary develops an internal wave in the thermocline resembling a soliton (Fig. 11). Combining the horizontal velocities at the sea surface from the numerical model with a radar imaging model, the internal wave signature can be observed in the radar backscatter intensity map of the sea surface (Fig. 12).

This internal wave also produces a strong signal in the induced magnetic field, reaching several $\mathrm{nT}$ at the leading edge of the wave (Figs. 13 and 14). This magnetic field signature is well within the range detectable by modern magnetometers.

Note that the internal wave signature is clearly seen in the velocity field, the magnetic field, as well as the simulated radar image. This suggests that data from magnetometers, oceanographic instruments and SAR satellites can complement each other to provide a comprehensive picture of dynamic processes in the upper ocean.

\section{Summary and discussion}

The approach used in this work, of combining a threedimensional CFD model, including an electromagnetic module, with a radar imaging model, can be useful for the simulation of fine-scale features on the sea surface and the interpretation of such signatures in high-resolution SAR images. We were able to reproduce the observed characteristics of sea surface signatures of centerline wakes of ships, surface buoyancy-driven currents and internal waves in SAR. The results are qualitatively consistent with available SAR images of ship wakes, freshwater plumes and internal waves. In the case of a thermal stratification in the oceanic near-surface layer, colder subsurface water may be brought to the surface through the circulation in the ship wake, and the stratification affects the signature of the wake at the surface by limiting the downward spreading of the wake. The model of a lowdensity near-surface buoyancy-driven current revealed that, as the density current propagates into a stratified environment, the interaction with the ambient stratification can lead to internal waves and a fragmentation of the surface plume. This fragmentation can be seen as alternating bands in the sea surface signature in simulated radar images. Internal waves 
can produce prominent sea surface signatures in SAR and can also lead to a signal in the measured electromagnetic field in the ocean. We reproduced the electromagnetic signatures of internal waves, low-density buoyancy-driven currents and ship wakes in the magnetic field.

The results presented in this work provide a link between SAR signatures of transient ocean dynamics and magnetic field fluctuations in the ocean. This suggests that combining SAR imagery with data from magnetometers may offer a valuable new prospect in maritime sensing.

Acknowledgements. This work was supported by the project "Hydrodynamics and Remote Sensing of Far Wakes of Ships" at NSUOC, by ONR Award N00014-10-1-0938 "Characterization of Impact of Oceanographic Features on the Electromagnetic Fields in Coastal Waters", and by the Gulf of Mexico Research Initiative Consortium for Advanced Research on the Transport of Hydrocarbons in the Environment. We are grateful to Richard Dodge (NSUOC) and Will Avera (NRLSSC) for helpful discussions of the problem. We thank Mikhail Gilman for help with programming user-defined functions for CFD.

Edited by: E. J. M. Delhez

\section{References}

Alpers, W.: Theory of radar imaging of internal waves, Nature, 314, 245-247, 1985.

Alpers, W., Brandt, P., and Rubino, A.: Internal waves generated in the Straits of Gibraltar and Messina: Observations from space, in: Remote Sensing of the European Seas, Springer, Heidelberg, 319-330, 2008.

Baines, P. G.: Mixing in flows down gentle slopes into stratified environments, J. Fluid Mech., 443, 237-270, 2001.

Beal, H. T. and Weaver, J. T.: Calculations of Magnetic Variations Induced by Internal Ocean Waves, J. Geophys. Res., 75, 68466852, 1970.

Benjamin, T. B.: Gravity currents and related phenomena, J. Fluid Mech., 31, 209-248, 1968.

Brandt, P., Romeiser, R., and Rubino, A.: On the determination of characteristics of the interior ocean dynamics from radar signatures of internal solitary waves, J. Geophys. Res., 104, 3003930045, 1999.

Brusch, S., Lehner, S., and Reppucci, A.: SAR Derived Wind Fields of Mesoscale Cyclones, Geoscience and Remote Sensing Symposium, IGARSS 2008 IEEE International, 2, 489-492, 2008.

Chave, A. D.: The magnetic effects of shallow water internal solitons, SIO Reference 86-7, 48 pp., Scripps Institution of Oceanography, La Jolla, 1986.

Crews, A. and Futterman, J.: Geomagnetic Micropulsations Due to the Motion of Ocean Waves, J. Geophys. Res., 67, 299-306, 1962.

Fujimura, A., Soloviev, A., and Kudryavtsev, V.: Numerical Simulation of the Wind-Stress Effect on SAR Imagery of Far Wakes of Ships, IEEE Geosci. Remote S., 7, 646-649, 2010.

Fujimura, A., Matt, S., Soloviev, A., Maingot, C., and Rhee, S. H.: The impact of thermal stratification and wind stress on sea surface features in SAR imagery, 2011 IEEE International Geoscience \& Remote Sensing Symposium (IGARSS) Proceedings, 2037-2040, 2011.

Gade, M., Alpers, W., Hühnerfuss, H., Masuko, H., and Kobayashi, T.: Imaging of biogenic and anthropogenic ocean surface films by the multifrequency/multipolarization SIR-C/X-SAR, J. Geophys. Res., 103, 18851-18866, 1998.

Grimshaw, W. R. and Yi, Z.: Resonant generation of finiteamplitude waves by the flow of a uniformly stratified fluid over topography, J. Fluid Mech., 229, 603-628, 1991.

Hennings, I., Romeiser, R., Alpers, W., and Viola, A.: Radar imaging of Kelvin arms of ship wakes, Int. J. Remote Sens., 20, 25192543, 1999.

Hinze, J. O.: Turbulence, McGraw-Hill, New York, 1975.

Horstmann, J., Koch, W., Lehner, S., and Tonboe R.: Wind Retrieval over the Ocean using Synthetic Aperture Radar with C-band HH Polarization, IEEE T. Geosci. Remote, 38, 2122-2131, 2000.

Hsu, M.-K., Liu, A. K., and Liu, C.: A study of internal waves in the China Seas and Yellow Sea using SAR, Cont. Shelf Res., 20, 389-410, 2000.

Hühnerfuss, H., Walter, W., Lange, P. A., and Alpers, W.: Attenuation of wind waves by monomolecular sea slicks and the Marangoni effect, J. Geophys. Res., 92, 3961-3963, 1987.

Issa, R. I.: Solution of the Implicit Discretized Fluid Flow Equations by Operator Splitting, Mechanical Engineering Rep. FS-82-15, Imperial College, London, 1982.

Lesieur, M.: Turbulence in Fluids, Springer, 596 pp., 2008.

Matt, S., Fujimura, A., Soloviev, A., and Rhee, S. H.: Modification of Turbulence at the Air-Sea Interface Due to the Presence of Surfactants and Implications for Gas Exchange. Part II: Numerical Simulations, in: Gas transfer at water surfaces 2010, Kyoto University Press, 299-312, 2011.

Milgram, J. H., Peltzer, R. D., and Griffin, O. M.: Suppression of short sea waves in ship wakes: measurements and observations, J. Geophys. Res., 98, 7103-7114, 1993a.

Milgram, J. H., Skop, R. A., Peltzer, R. D., and Griffin, O. M.: Modeling short sea wave energy distribution in the far wakes of ships, J. Geophys. Res., 98, 7115-8370, 1993 b.

Nash, J. D. and Moum, J. N.: River plumes as a source of largeamplitude internal waves in the coastal ocean, Nature, 437, 400403, 2005.

Nicoud, F. and Ducros, F.: Subgrid-scale stress modelling based on the square of the velocity gradient tensor, Flow, Turbulence and Combustion, 62, 183-200, 1999.

Patankar, S. V.: Numerical Heat Transfer and Fluid Flow, Hemisphere, Washington, DC, 214 pp., 1980.

Pichel, W. G., Clemente-Colon, P., Wackerman, C. C., and Friedman, K. S.: Ship and wake detection, in: Synthetic Aperture Radar Marine User's Manual, edited by: Jackson, C. R. and Apel, J. R., NOAA, 277-303, 2004.

Reed, A. M. and Milgram, J. H.: Ship wakes and their radar images, Annu. Rev. Fluid Mech., 34, 469-502, 2002.

Rhee, S. H. and Kim, H.: A suggestion of gap flow control devices for the suppression of rudder cavitation, J. Mar. Sci. Technol., 13, 356-370, 2008.

Romeiser, R.: M4S 3.2.0 User's Manual, University of Hamburg, Hamburg, Germany, 2008.

Romeiser, R. and Alpers, W.: An improved composite surface model for the radar backscattering cross section of the ocean 
surface, 2. Model response to surface roughness variations and the radar imaging of underwater bottom topography, J. Geophys. Res., 102, 25251-25267, 1997.

Romeiser, R., Alpers, W., and Wismann, V.: An improved composite surface model for the radar backscattering cross section of the ocean surface, 1 . Theory of the model and optimization validation by scatterometer data, J. Geophys. Res., 102, 25237-25250, 1997.

Romeiser, R., Ufermann, S., and Kern, S.: Remote sensing of oceanic current features by synthetic aperture radar - achievements and perspectives, Ann. Telecommun., 56, 661-671, 2001.

Romeiser, R., Suchandt, S., Runge, H., Steinbrecher, U., and Grünler, S.: First analysis of TerraSAR-X along-track InSAR-derived current fields, IEEE T. Geosci. Remote, 48, 820-829, 2010.

Sagaut, P.: Large Eddy Simulation for Incrompressible Flows, An Introduction, Springer, 1998.

Simpson, J. E.: Gravity Currents: In the Environment and the Laboratory, Ellis Horwood Limited, 244 pp., 1987.

Smagorinsky, J.: General Circulation Experiments with the Primitive Equations. I. The Basic Experiment, Mon. Weather Rev., 91, 99-164, 1963.

Soloviev, A. V. and Lukas, R.: Sharp frontal interfaces in nearsurface layer of the ocean in the western equatorial Pacific warm pool, J. Phys. Oceanogr., 27, 999-1017, 1997.

Soloviev, A. and Lukas, R.: The Near-Surface Layer of the Ocean: Structure, Dynamics, and Applications, Springer, New York, 572 pp., 2006.
Soloviev, A., Gilman, M., Moore, K., Young, K., and Graber, H.: Hydrodynamics and Remote Sensing of Far Wakes of Ships, SEASAR 2008 - The 2nd Int. Workshop on Advances in SAR Oceanography, Frascati, Italy, 21-25 January, 2008.

Soloviev, A., Gilman, M., Young, K., Brusch, S., and Lehner, S.: Sonar measurements in ship wakes simultaneous with TerraSARX overpasses, IEEE T. Geosci. Remote, 48, 841-851, 2010.

Soloviev, A., Maingot, C., Matt, S., Dodge, R. E., Lehner, S., Velotto, D., Brusch, S., Perrie, W., and Hochberg, E.: Fine-scale features on the sea surface in SAR satellite imagery - Part 1: Simultaneous in-situ measurements, Ocean Sci. Discuss., 9, 2885 2914, doi:10.5194/osd-9-2885-2012, 2012.

Soloviev, A., Matt, S., and Avera, W.: Analysis of the electromagnetic signatures of fine-scale oceanographic features, Proceedings of MARELEC 2013, 16-19 July, Hamburg, Germany, 2013.

Toporkov, J. V., Hwang, P. A., Sletten, M. A., Farquharson, G., Perkovic, D., and Frasier, S. J.: Surface Velocity Profiles in a Vessel's Turbulent Wake Observed by a Dual-Beam Along-Track Interferometric SAR, IEEE Geosci. Remote S., 8, 602-606, 2011.

Ufermann, S. and Romeiser, R.: A new interpretation of multifrequency/multipolarization radar signatures of the Gulf Stream front, J. Geophys. Res., 104, 25697-25706, 1999.

White, B. L. and Helfrich, K. R.: A general description of a gravity current front propagating into a two-layer stratified fluid, J. Fluid. Mech., 711, 545-575, 2012. 